\title{
INTERPRETAÇÃO DE RESULTADOS ANALÍTICOS DE FÓSFORO PELOS EXTRATORES MEHLICH-1 E MEHLICH-3 EM SOLOS DO RIO GRANDE DO SUL ${ }^{(1)}$
}

\author{
Leandro Bortolon $^{(2)} \&$ Clesio Gianello ${ }^{(3)}$
}

\begin{abstract}
RESUMO
As soluções extratoras multielementares possibilitam avaliar a disponibilidade de vários nutrientes de plantas no mesmo extrato; dentre estes o P merece especial atenção. As soluções Mehlich-1 ( $\left.M_{1}\right)$ e Mehlich-3 $\left(M_{3}\right)$ enquadram-se nesta categoria. Neste estudo, foram comparados os teores de P extraídos por estas soluções em 360 amostras de solos do Estado do Rio Grande do Sul. Os coeficientes de correlação entre os teores de $\mathrm{P}$ extraído pelas mesmas indicaram alto grau de associação e de significância. Os teores de $\mathrm{P}$ extraído pela solução Mehlich-3 foram, em média, 50 \% maiores que os extraídos pela solução Mehlich-1. O teor de argila, entretanto, influenciou a capacidade extrativa de $\mathbf{P}$ pela solução Mehlich-3, sendo necessária uma tabela de ajuste para a interpretação dos valores do P extraído por esta solução.
\end{abstract}

Termos de indexação: análise de solo, Mehlich-1, Mehlich-3.

SUMMARY: INTERPRETATION OF THE AVALILABRE PHOSPHORUS BY MEHLICH-1 AND MEHLICH-3 EXTRACTANT SOLUTION IN SOILS OF THE RIO GRANDE DO SUL STATE, BRAZIL

Multielement soil extractant solutions are used by several soil testing laboratories to determine the plant availability of several nutrients by the same extractant, to decrease costs

\footnotetext{
(1) Parte da Dissertação de Mestrado do primeiro autor, apresentada ao Programa de Pós-Graduação em Ciência do Solo, Universidade Federal do Rio Grande do Sul - UFRGS. Executada com recursos do projeto Laboratório de Análises do Departamento de Solos da Faculdade de Agronomia - UFRGS. Trabalho apresentado no XXXI Congresso Brasileiro de Ciência do Solo, 2007 (Gramado, RS).

(2) Pós-graduando em Ciência do Solo da Universidade Federal do Rio Grande do Sul - UFRGS. Av. Bento Gonçalves, 7712 . CEP 91540-000, Porto Alegre (RS). Bolsista do CNPq. E-mail: leandro.bortolon@ufrgs.br

(3) Professor Associado do Departamento de Solos, UFRGS. E-mail: cgianello@hotmail.com.br
} 
and labor. The Mehlich-1 $\left(M_{1}\right)$ and Mehlich-3 $\left(M_{3}\right)$ solutions are often used for this purpose. In this study, they were used to evaluate soil $P$ availability using 360 soil samples of the State of Rio Grande do Sul (Southern Brazil). The correlation coefficients between the P concentrations extracted by these solutions were strongly associated and highly significant. The $P$ concentrations extracted by the $M_{3}$ solution were on average $50 \%$ higher than those extracted by $M_{1}$. The soil clay content affected the $P$ extraction capacity of the $M_{3}$ solution, requiring an adjustment table for the interpretation of the $P$ value by this method, as proposed here.

Index terms: soil test, Mehlich-1, Mehlich-3.

\section{INTRODUÇÃO}

A utilização de soluções extratoras multielementares está sendo estudada por diversos pesquisadores com o objetivo de simplificar os procedimentos analíticos, reduzir o custo das análises e testar a extração simultânea de vários nutrientes de plantas. Concomitantemente, a espectrometria de emissão ótica em plasma induzido (ICP-OES) está sendo utilizada em alguns laboratórios, para aumentar a exatidão analítica, diminuir os limites de detecção e determinar concomitantemente vários elementos (Milagres et al., 2007).

A solução extratora Mehlich-3 $\left(\mathrm{M}_{3}\right)$ tem sido uma das mais estudadas para essa finalidade (Raij, 1994); entretanto, para sua utilização nos laboratórios de análise de solo, deve ser feita a calibração dos valores obtidos pelo método com o rendimento em culturas numa determinada região. No Estado do RS, foi realizado um estudo de calibração dessa solução para $\mathrm{P} \mathrm{e} \mathrm{K}$, em que foi observada sua adequabilidade para avaliar a disponibilidade desses nutrientes às plantas (Schlindwein \& Gianello, 2006). Entretanto, esse estudo de calibração foi feito para o sistema plantio direto.

A possibilidade de uso da solução $\mathrm{M}_{3}$ para a extração de outros elementos foi pouco estudada em solos do RS. Estudo feito por Bortolon (2005) indicou que essa solução pode ser utilizada para extração simultânea de $\mathrm{P}, \mathrm{K}$, $\mathrm{Cu}, \mathrm{Zn}, \mathrm{Ca}$ e $\mathrm{Mg}$, sendo viável sua utilização em laboratórios de análises de solo. Além disso, outros estudos feitos no RS comprovam a eficiência dessa solução para a extração de P (Kroth, 1998; Schlindwein, 2003; Bortolon \& Gianello 2006). Essa constatação indica que a solução $\mathrm{M}_{3}$ pode substituir as soluções extratoras atualmente em uso, conforme sugerido em diversos trabalhos (Wolf \& Baker, 1985; Jones \& Piha, 1989; Sims, 1989; Mamo et al., 1996; Eckert \& Watson, 1997; Garcia et al., 1997). Os diversos trabalhos publicados não relacionam a capacidade extrativa das soluções $\mathrm{M}_{1}$ e $\mathrm{M}_{3}$ com atributos físicos e químicos do solo. Entretanto, a variabilidade dos solos do sul do Brasil altera a dinâmica do $\mathrm{P}$ no solo e a capacidade extrativa da solução $\mathrm{M}_{1}$, requerendo a determinação do teor de argila para a interpretação dos teores de $\mathrm{P}$ no solo (CQFSRS/SC, 2004). O mesmo ocorre em outras regiões do País (Minas Gerais, Cerrados, Mato Grosso,
Paraná), onde essa solução é utilizada para extração de $\mathrm{P}$ do solo.

O presente trabalho teve por objetivo determinar a capacidade extrativa de $\mathrm{P}$ das soluções de Mehlich1 e de Mehlich-3, relacionando-as com as classes de argila, e propor uma tabela de interpretação dos teores de P de solos do Rio Grande do Sul, extraídos pela solução de Mehlich-3.

\section{MATERIAL E MÉTODOS}

O estudo foi realizado no Laboratório de Análises de Solo da UFRGS, com 360 amostras de solos das classes Latossolo, Argissolo, Cambissolo, Planossolo, Neossolo, Nitossolo, Vertissolo e Chernossolo, provenientes do RS. Algumas dessas amostras foram anteriormente utilizadas em outros estudos de disponibilidade de nutrientes (Miola, 1995; Kroth, 1998; Bortolon, 2005).

O P dos solos foi extraído pela solução de Mehlich-1 $\left(\mathrm{M}_{1}\right)\left(\mathrm{HCl} \mathrm{0,05} \mathrm{mol} \mathrm{L}^{-1}+\mathrm{H}_{2} \mathrm{SO}_{4} 0,0125 \mathrm{~mol} \mathrm{~L}^{-1}\right) ; \mathrm{pH}$ $1,2)$ na relação solo:solução de 1:10, com agitação por 5 min em agitador horizontal a 120 oscilações por minuto e decantação por $16 \mathrm{~h}$, conforme método descrito por Tedesco et al. (1995). Esse nutriente foi também extraído pela solução de Mehlich-3 $\left(\mathrm{M}_{3}\right)\left(\mathrm{CH}_{3} \mathrm{COOH}\right.$ $0,2 \mathrm{~mol} \mathrm{~L}^{-1}+\mathrm{NH}_{4} \mathrm{NO}_{3} 0,25 \mathrm{~mol} \mathrm{~L}^{-1}+\mathrm{NH}_{4} \mathrm{~F} 0,015$ mol L-1 + $\mathrm{HNO}_{3} 0,013 \mathrm{~mol} \mathrm{~L}^{-1}+$ EDTA $0,001 \mathrm{~mol} \mathrm{~L}^{-1}$; pH 2,5), na relação solo:solução de 1:10, com agitação por 5 min em agitador orbital a 220 rotações por minuto. A filtração dos extratos, proposta por Mehlich (1984), foi substituída por decantação por 16 h. Nos extratos, o $\mathrm{P}$ foi determinado por colorimetria (Tedesco et al., 1995).

Foi feita a análise de correlação entre os teores de $\mathrm{P}$ extraído pelas soluções e as equações foram ajustadas pelo modelo linear.

\section{RESULTADOS E DISCUSSÃO}

No quadro 1, são apresentados os teores (máximo, mínimo), a média e a mediana das diferentes determinações. Os valores obtidos situam-se nas faixas usualmente determinadas em solos agrícolas. 
Quadro 1. Amplitude dos valores de pH e dos teores de argila, matéria orgânica e P extraídos pelas soluções de Mehlich-1 e de Mehlich-3 dos solos ${ }^{1}$

\begin{tabular}{lccccc}
\hline \multirow{2}{*}{ Parâmetros } & \multirow{2}{*}{$\begin{array}{c}\text { pH } \\
\text { em água }\end{array}$} & Argila & $\begin{array}{c}\text { Matéria } \\
\text { Orgânica }\end{array}$ & \multicolumn{2}{c}{ P extraído } \\
\cline { 3 - 6 } & & & $\mathrm{g} \mathrm{dm}^{-3}$ & & $\mathrm{mg} \mathrm{dm}^{-3}$ \\
Mínimo & 4,8 & 30 & 5 & 0,5 & 0,1 \\
Máximo & 6,5 & 700 & 181 & 91,9 & 140,1 \\
Média & 5,6 & 300 & 30 & 15,0 & 21,9 \\
Mediana & 5,6 & 250 & 26 & 8,2 & 9,9 \\
\hline
\end{tabular}

(1)Determinações efetuadas conforme método descrito por Tedesco et al. (1995).

Os teores de $\mathrm{P}$ extraído do solo pelas soluções $\mathrm{M}_{1} \mathrm{e}$ $\mathrm{M}_{3}$ apresentaram alto grau de correlação $\left(\mathrm{r}=0,95^{* *}\right)$ (Figura 1). Em média, os teores extraídos pela solução $\mathrm{M}_{3}$ foram, aproximadamente, $50 \%$ maiores do que os extraídos pela solução $\mathrm{M}_{1}$. O maior teor de $\mathrm{P}$ extraído com a solução $\mathrm{M}_{3}$ deve-se, em parte, às características químicas da mesma, que extrai preferencialmente o $\mathrm{P}$ ligado ao $\mathrm{Fe}$ e ao $\mathrm{Al}$ e, em menor proporção, ao $\mathrm{P}$ ligado ao Ca (Beegle, 2005). O mesmo foi observado por Kroth (1998), com alguns solos deste estudo e por outros autores (Wolf \& Baker, 1985; Sims, 1989; Gartley et al., 2002; Gattiboni, 2003; Schlindwein, 2003; Ring et al., 2004).

Nos Estados do RS e de SC a interpretação dos teores do P no solo é feita com base no teor de argila. Isto ocorre pelo fato de o teor de argila alterar a

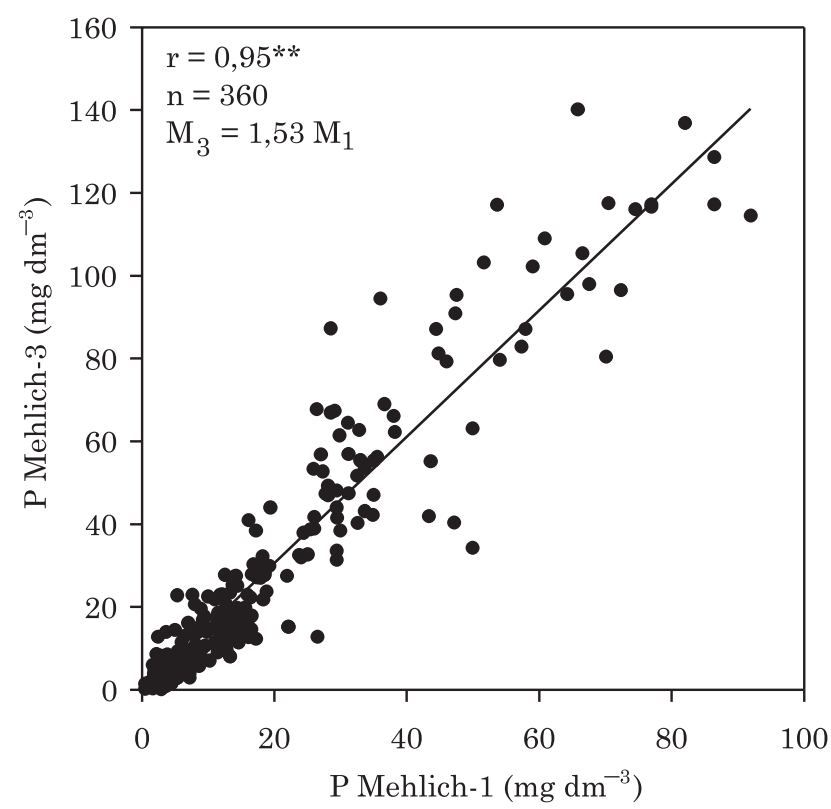

Figura 1. Correlação entre os teores de $\mathbf{P}$ extraído pelas soluções de Mehlich-1 e de Mehlich-3 em 360 amostras de solos do Rio Grande do Sul. capacidade extrativa da solução $\mathrm{M}_{1}$. A proposta para classificar os teores de argila para a interpretação dos teores de $\mathrm{P}$ do solo extraído pela solução de Mehlich-1, em solos do Rio Grande do Sul, foi feita por Anghinoni \& Bohnen (1974). Essa classificação é atualmente composta por quatro classes de argila, a saber: Classe 1 (teor de argila $>600 \mathrm{~g} \mathrm{dm}^{-3}$ ), Classe 2 (teores de argila entre 410 e $600 \mathrm{~g} \mathrm{dm}^{-3}$ ), Classe 3 (teores de argila entre 210 e $400 \mathrm{~g} \mathrm{dm}^{-3}$ ) e Classe 4 (teor de argila $\leq 200 \mathrm{~g} \mathrm{dm}^{-3}$ ). Nos diversos trabalhos publicados, não é mencionado o efeito do teor de argila sobre a capacidade extrativa da solução $\mathrm{M}_{3}$ (Wolf \& Baker, 1985; Sims, 1989; Gartley et al., 2002; Gattiboni, 2003; Schlindwein, 2003; Ring et al., 2004).

$\mathrm{Na}$ figura 2, são mostradas as correlações entre os teores de $\mathrm{P}$ extraído pelas soluções, em solos com teores de argila $\leq 200 \mathrm{~g} \mathrm{dm}^{-3}$ (a) e entre 210 e $400 \mathrm{~g}$ $\mathrm{dm}^{-3}$ (b), e teores de $\mathrm{P}$ menores que $50 \mathrm{mg} \mathrm{dm}^{-3} \mathrm{e}$ $40 \mathrm{mg} \mathrm{dm}^{-3}$, respectivamente, extraídos pela solução $\mathrm{M}_{1}$. Foi observado que, em solos da classe $4(\leq 200 \mathrm{~g}$ $\mathrm{dm}^{-3}$ ), a solução $\mathrm{M}_{3}$ extraiu, em média, $60 \%$ a mais de $\mathrm{P}$ em relação à solução $\mathrm{M}_{1}$. Para os solos da classe 3 (teor de argila entre 210 e $400 \mathrm{~g} \mathrm{dm}^{-3}$ ), a solução $\mathrm{M}_{3}$ extraiu, em média, $50 \%$ a mais de $\mathrm{P}$ do que a solução $M_{1}$. Nos solos da classe 2 (com teores de argila entre 410 e $600 \mathrm{~g} \mathrm{dm}^{-3}$ ) e nos da classe $1\left(>600 \mathrm{~g} \mathrm{dm}^{-3}\right)$, que apresentaram teores de $\mathrm{P}$ menores do que $20 \mathrm{mg} \mathrm{dm}^{-3}$ e $10 \mathrm{mg} \mathrm{dm}^{-3}$, respectivamente, a solução $\mathrm{M}_{3}$ extraiu menos $\mathrm{P}$ em relação às demais classes (Figura 3 ). Nos solos da classe 2, os teores de P extraído foram semelhantes; entretanto, nos solos da classe 1 , os teores de P extraído pela solução $\mathrm{M}_{3}$, foram aproximadamente, 20 \% menores que os extraídos pela solução $\mathrm{M}_{1}$. A utilização de uma simples equação de conversão para todos os solos dos Estados do RS e de SC, portanto, não é viável, em virtude de variações na capacidade extrativa de $\mathrm{P}$ da solução $\mathrm{M}_{3} \operatorname{com} \mathrm{o}$ aumento do teor de argila do solo. Neste caso, é necessário o ajuste de valores de $\mathrm{P}$ extraído pela solução $\mathrm{M}_{3}$, para cada classe de solo, estabelecendose uma tabela de interpretação de valores analíticos de $\mathrm{P}$ extraído pela mesma. 

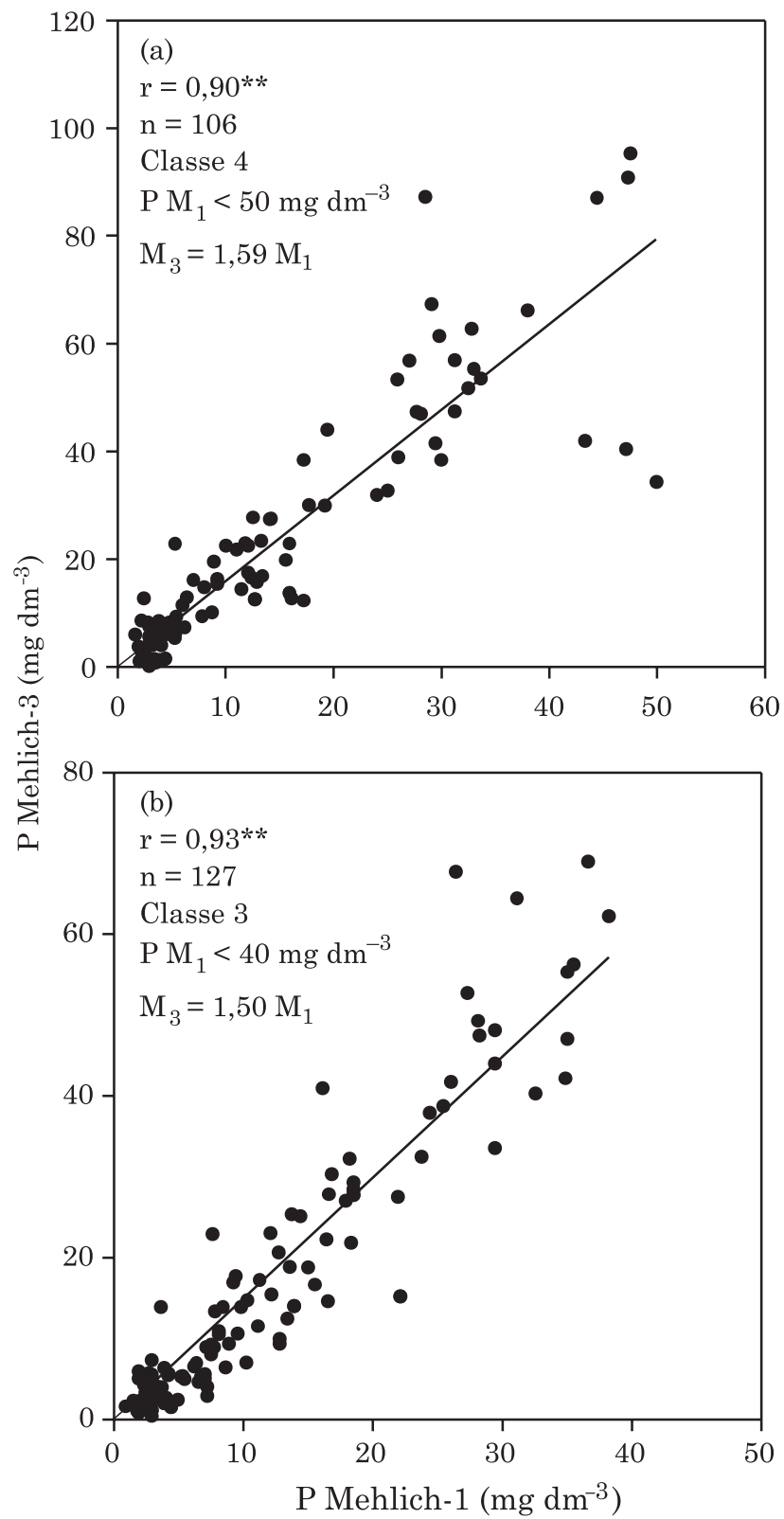

Figura 2. Correlações entre os teores de P extraído pelas soluções de Mehlich-1 e de Mehlich-3, para solos com teores de argila $=200 \mathrm{~g} \mathrm{dm}^{-3}$ (Classe 4) (a) e entre 210 e $400 \mathrm{~g} \mathrm{dm}^{-3}$ (Classe 3) (b).

Neste trabalho, foram propostas faixas de interpretação dos teores de P no solo extraído pela solução de Mehlich-3. As equações utilizadas para a elaboração da tabela foram ajustadas pelo modelo linear, passando pela origem. As equações foram feitas excluindo-se solos, dentro de cada classe de argila, com teores de P maiores do que os da classe "Alto". As equações utilizadas para cada classe foram as especificadas nas figuras 2 e 3 . Para solos alagados, como não ocorre interferência dos teores de argila nos do $\mathrm{P}$, foi utilizada a equação de ajuste estabelecida para todos os solos (Figura 1). A proposta
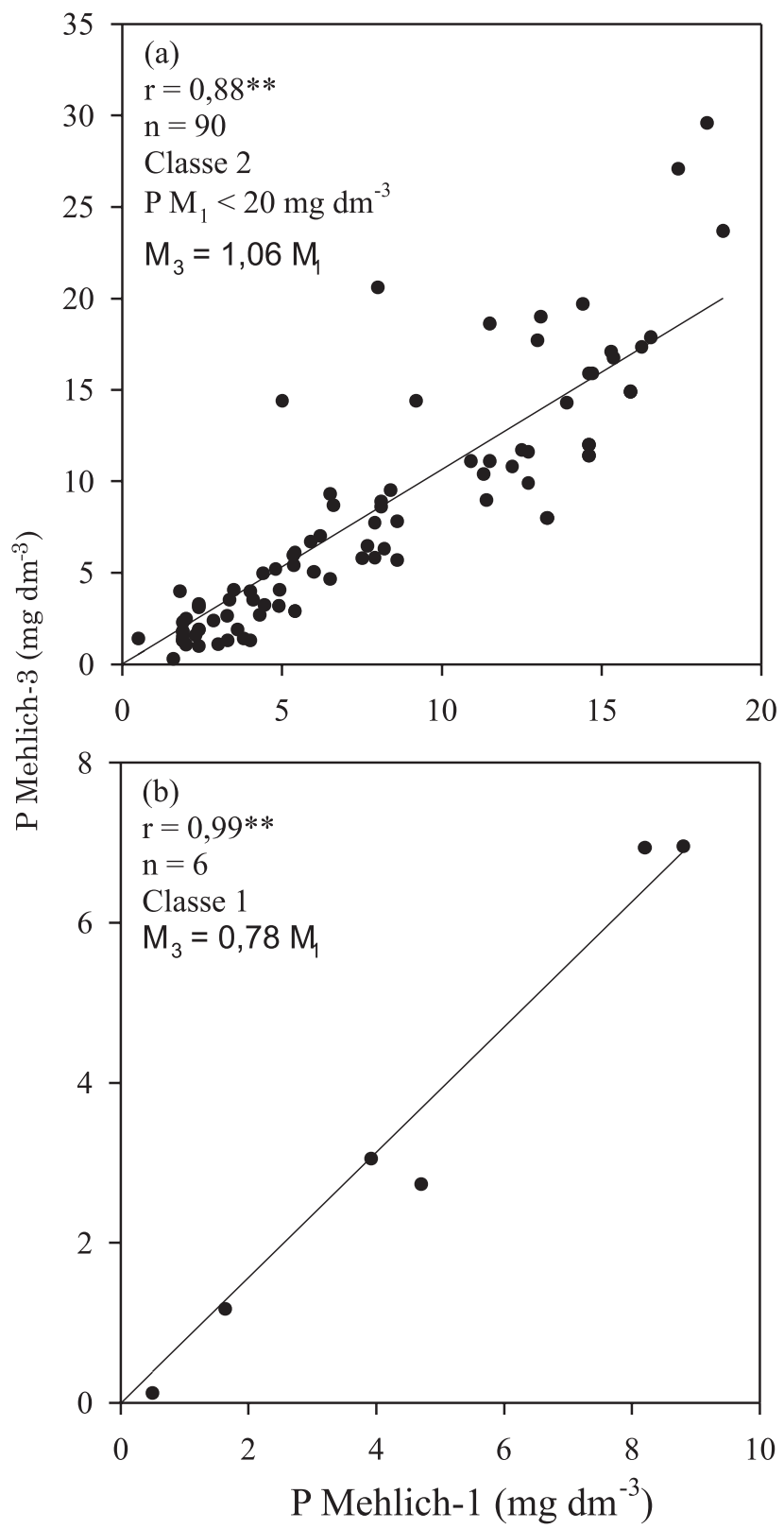

Figura 3. Correlações entre os teores de P extraído pelas soluções de Mehlich-1 e de Mehlich-3, para solos com teores de argila entre 410 e $600 \mathrm{~g} \mathrm{dm}^{-3}$ (Classe 2) (a) e $>600 \mathrm{~g} \mathrm{dm}^{-3}$ (Classe 1) (b).

de faixas de interpretação dos teores de $\mathrm{P}$ no solo, extraído pela solução de Mehlich-3, é mostrada no quadro 2.

\section{CONCLUSÕES}

1. Os extratores Mehlich-1 e Mehlich-3 são adequados para a extração de $\mathrm{P}$, podendo ser utilizados em laboratórios de análise para a finalidade de diagnóstico da fertilidade do solo. 
Quadro 2. Interpretação dos teores de P do solo extraído pela solução de Mehlich-3 conforme a classe textural

\begin{tabular}{|c|c|c|c|c|c|}
\hline \multirow{2}{*}{$\begin{array}{l}\text { Faixas de } \\
\text { Interpretação }\end{array}$} & \multicolumn{4}{|c|}{ Classe textural do solo(1) } & \multirow{2}{*}{$\begin{array}{c}\text { Solos } \\
\text { Alagados }\end{array}$} \\
\hline & 1 & 2 & 3 & 4 & \\
\hline & & & $\mathrm{mg} \mathrm{dm} \mathrm{m}^{-3}$ & & \\
\hline Muito Baixo & $\leq 1,5$ & $\leq 3,0$ & $\leq 5,5$ & $\leq 10,0$ & - \\
\hline Baixo & $1,6-3,0$ & $3,1-6,0$ & $5,6-11,0$ & $10,1-20,0$ & $\leq 4,0$ \\
\hline Médio(2) & $3,1-5,0$ & $6,1-10,0$ & $11,1-18,0$ & $20,1-34,0$ & $4,1-8,0$ \\
\hline Alto & $5,1-10,0$ & $10,1-20,0$ & $18,1-36,0$ & $34,1-68,0$ & $8,1-16,0$ \\
\hline Muito Alto $^{(3)}$ & $>10,0$ & $>20,0$ & $>36,0$ & $>68,0$ & $>16,0$ \\
\hline
\end{tabular}

2. O aumento do teor de argila reduziu a capacidade extrativa da solução de Mehlich-3 em relação à de Mehlich-1, sendo necessária uma tabela de interpretação dos teores de $\mathrm{P}$ do solo adaptada para este extrator.

\section{LITERATURA CITADA}

ANGHINONI, I. \& BOHNEN, H. Avaliação da disponibilidade de fósforo através de métodos químicos. Agron. Sulriograndense, 10:127-133, 1974.

BEEGLE, D. Assesing soil phosphorus for crop production by soil testing. In: SIMS J.T. e SHARPLEY, A.N., eds. Phosphorus: Agriculture and the environment. Modison, Soil Science Society of America, 2005, p.123-144.

BORTOLON, L. Métodos de avaliação da disponibilidade de nutrientes para as plantas em solos do Rio Grande do Sul. Porto Alegre, Universidade Federal do Rio Grande do Sul, 2005. 111p. (Tese de Mestrado)

BORTOLON, L. \& GIANELLO, C. Phosphorus availability to maize determined by several methods on Rio Grande do Sul (Brazil) soils. In: INTERNATIONAL SYMPOSIUM PHOSPHORUS DYNAMICS IN SOIL-PLANT CONTINUUM, 3., Uberlândia, 2006. Anais. Uberlândia, Embrapa, 2006. p.49-52.

COMISSÃO DE QUIMICA E FERTILIDADE DO SOLO CQFSRS/SC. Manual de adubação e de calagem para os Estados do Rio Grande do Sul e Santa Catarina. 10.ed. Porto Alegre, Sociedade Brasileira de Ciência do Solo/ Núcleo Regional Sul, 2004. 400p.

ECKERT, D.J. \& WATSON, M.E. Integrating the Mehlich-3 extractant into existing soil test interpretation schemes. In: HOOD, T.M \& JONES, J.B. Soil and plant analysis in sustainable agriculture and environment. New York: Marcel Dekker, 1997. p.239-251.
GARCIA, A.; IORIO, A.F.; BARROS, M.; BARGIELA, M. \& RENDINA, A. Comparision of soil tests to determinate micronutrients status in Argentina soils. Comm. Soil Sci. Plant Anal., 28:1777-1792, 1997.

GARTLEY, K.L.; SIMS, J.T.; OLSEN, C.T. \& CHU, P. Comparision of soil test extractants used in Mid-Atlantic United States. Comm. Soil Sci. Plant Anal., 33:873-895, 2002.

GATTIBONI, L.C. Disponibilidade de formas de fósforo do solo às plantas. Santa Maria, Universidade Federal de Santa Maria, 2003. 213p. (Tese de Doutorado)

JONES, U.S. \& PIHA, M. Evaluation of four tests extractants for Zimbabwe soils. Comm. Soil Sci. Plant Anal., 20:1857$1871,1989$.

KROTH, P.L. Disponibilidade de fósforo no solo para as plantas e fatores que afetam a extração por resina de troca em membranas. Porto Alegre, Universidade Federal do Rio Grande do Sul, 1998. 167p. (Tese de Mestrado)

MAMO, T.; RICHTER, C. \& HEILIGTAG, B. Comparision of extractants for the determination of available phosphorus, potassium, calcium, magnesium and sodium in some Ethiopian and German soils. Comm. Soil Sci. Plant Anal., 27:2197-2212, 1996.

MEHLICH, A. Mehlich 3 soil test extractant: A modification of Mehlich 2 extractant. Comm. Soil Sci. Plant Anal., 15:14091416. 1984.

MILAGRES, J.J.M.; ALVAREZ V., V.H.; NEVES, J.C.L.; CANTARUTTI, R.B. \& BORGES Jr., M. Determinação de $\mathrm{Fe}, \mathrm{Zn}, \mathrm{Cu}$ e $\mathrm{Mn}$ extraídos do solo por diferentes extratores e dosados por espectrofotometria de emissão ótica em plasma induzido e espectrofotometria de absorção atômica. R. Bras. Ci. Solo, 31:237245. 2007. 
MIOLA, G.R. Extração de P, K, Ca e Mg do solo por diferentes métodos e avaliação da disponibilidade de fósforo para as plantas. Porto Alegre, Universidade Federal do Rio Grande do Sul, 1995. 127p. (Tese de Mestrado)

RAIJ, B.van. New diagnostics techniques, universal soil extractants. Comm. Soil Sci. Plant Anal., 25:799-816, 1994.

RING, R.A.; WARMAN, P.R.; STRATTON, G.W. \& EATON, L.J. Determining available soil phosphorus in Nova Scotia Blueberry Soils. Comm. Soil Sci. Plant Anal., 35:2449-2463, 2004.

SCHLINDWEIN, J.A. Calibração de métodos de determinação e estimativa de doses de fósforo e potássio em solos sob sistema plantio direto. Porto Alegre, Universidade Federal do Rio Grande do Sul, 2003. 169p. (Tese de Doutorado)
SCHLINDWEIN, J.A. \& GIANELLO, C. Recomendações de fertilizantes no RS e o impacto na fertilidade do solo e no rendimento das culturas. R. Plantio Direto, 91:17-25, 2006.

SIMS, J.T. Comparison of Mehlich 1 and Mehlich 3 extractants for P, K, Ca, Mg, Mn, Cu and $\mathrm{Zn}$ in Atlantic Coastal Plain soils. Comm. Soil Sci. Plant Anal., 20:1707-1726, 1989.

TEDESCO, M.J.; GIANELLO, C.; BISSANI, C.A.; BOHNEN, H. \& VOLKWEISS, S.J. Análise de solo, plantas e outros materiais. 2.ed. Porto Alegre, Universidade Federal do Rio Grande do Sul, 1995. 147p. (Boletim Técnico, 5)

WOLF, A.M. \& BAKER, D.E. Comparisons of soil test phosphorus by Olsen, Bray 1, Mehlich 1 and Mehlich 3 methods. Comm. Soil Sci. Plant Anal., 16:467-484, 1985. 